\title{
EXTREMES OF HOMOGENEOUS GAUSSIAN RANDOM FIELDS
}

\author{
KRZYSZTOF DȨBICKI, ${ }^{*}$ University of Wroctaw \\ ENKELEJD HASHORVA, ${ }^{* *}$ University of Lausanne \\ NATALIA SOJA-KUKIEłA, ${ }^{* * *}$ Nicolaus Copernicus University
}

\begin{abstract}
Let $\{X(s, t): s, t \geq 0\}$ be a centred homogeneous Gaussian field with almost surely continuous sample paths and correlation function $r(s, t)=\operatorname{cov}(X(s, t), X(0,0))$ such that $r(s, t)=1-|s|^{\alpha_{1}}-|t|^{\alpha_{2}}+o\left(|s|^{\alpha_{1}}+|t|^{\alpha_{2}}\right), s, t \rightarrow 0$, with $\alpha_{1}, \alpha_{2} \in(0,2]$, and $r(s, t)<1$ for $(s, t) \neq(0,0)$. In this contribution we derive an asymptotic expansion (as $u \rightarrow \infty)$ of $\mathbb{P}\left(\sup _{\left(s n_{1}(u), t n_{2}(u)\right) \in[0, x] \times[0, y]} X(s, t) \leq u\right)$, where $n_{1}(u) n_{2}(u)=$ $u^{2 / \alpha_{1}+2 / \alpha_{2}} \Psi(u)$, which holds uniformly for $(x, y) \in[A, B]^{2}$ with $A, B$ two positive constants and $\Psi$ the survival function of an $N(0,1)$ random variable. We apply our findings to the analysis of extremes of homogeneous Gaussian fields over more complex parameter sets and a ball of random radius. Additionally, we determine the extremal index of the discretised random field determined by $X(s, t)$.
\end{abstract}

Keywords: Gaussian random field; supremum; tail asymptoticy; extremal index; Berman condition; strong dependence

2010 Mathematics Subject Classification: Primary 60G15

Secondary $60 \mathrm{G} 70$

\section{Introduction}

One of the seminal results in extreme value theory of Gaussian processes is the asymptotic behaviour of the distribution of the supremum of a centred stationary Gaussian process $\{X(t): t \geq 0\}$ with correlation function satisfying

$$
r(t)=\operatorname{cov}(X(t), X(0))=1-|t|^{\alpha}+o\left(|t|^{\alpha}\right) \quad \text { as } t \rightarrow 0 \text { with } \alpha \in(0,2],
$$

over intervals of length proportional to

$$
\mu(u)=\mathbb{P}\left(\sup _{t \in[0,1]} X(t)>u\right)^{-1}(1+o(1))
$$

see, e.g. Leadbetter et al. [1, Theorem 12.3.4], Arendarczyk and Dȩbicki [2, Lemma 4.3], and Tan and Hashorva [3, Lemma 3.3]. The following theorem gives a preliminary result concerning the aforementioned asymptotics.

Received 17 April 2013; revision received 13 December 2013.

* Postal address: Mathematical Institute, University of Wrocław, pl. Grunwaldzki 2/4, 50-384 Wrocław, Poland. Email address: debicki@math.uni.wroc.pl

** Postal address: Faculty of Business and Economics (HEC Lausanne), University of Lausanne, 1015 Lausanne, Switzerland.

*** Postal address: Nicolaus Copernicus University, ul. Chopina 12/18, 87-100 Toruń, Poland. 
Theorem 1. Let $\{X(t): t \geq 0\}$ be a centred stationary Gaussian process that satisfies (1), and let $0<A<B<\infty$ and $x>0$ be arbitrary constants. If $r(t) \log t \rightarrow r \in[0, \infty)$ as $t \rightarrow \infty$, then

$$
\mathbb{P}\left(\sup _{t \in[0, x \mu(u)]} X(t) \leq u\right) \rightarrow \mathbb{E}(\exp (-x \exp (-r+\sqrt{2 r} w))) \in(0, \infty),
$$

as $u \rightarrow \infty$, uniformly for $x \in[A, B]$, with $\mathcal{W}$ an $N(0,1)$ random variable $(R V)$.

The main goal of this paper is to derive an analogue of the above result for Gaussian random fields; see part (i) of Theorem 2 which constitutes a two-dimensional counterpart of Theorem 1.

As an application of our findings, we investigate asymptotics of the tail of the supremum of a homogeneous Gaussian field over parameter sets that are approximable by simple sets (part (ii) of Theorem 2) and a ball of random radius. Additionally, we analyse the existence of the extremal index for discrete-parameter fields associated with homogeneous Gaussian fields with a covariance structure satisfying some regularity conditions; see Proposition 2.

\section{Preliminaries}

Let $\{X(s, t): s, t \geq 0\}$ be a centred homogeneous Gaussian field with almost surely (a.s.) continuous sample paths and correlation function $r(s, t)=\operatorname{cov}(X(s, t), X(0,0))$ such that

A1: $r(s, t)=1-|s|^{\alpha_{1}}-|t|^{\alpha_{2}}+o\left(|s|^{\alpha_{1}}+|t|^{\alpha_{2}}\right)$ as $s, t \rightarrow 0$ with $\alpha_{1}, \alpha_{2} \in(0,2]$;

A2: $r(s, t)<1$ for $(s, t) \neq(0,0)$;

A3: $\sup _{(s, t) \in \delta(0, d)}|r(s, t) \log d-r| \rightarrow 0$ as $d \rightarrow \infty$ with $r \in[0, \infty)$,

where $\delta(0, d)$ denotes the sphere of centre $(0,0)$ and radius $d>0$ in $\mathbb{R}^{2}$ with Euclidean metric.

We distinguish two separate families of Gaussian fields:

- weakly dependent fields, satisfying A3 with $r=0$,

- strongly dependent fields, satisfying A3 with $r \in(0, \infty)$.

Let $\mathscr{H}_{\alpha}$ denote the Pickands' constant (see [4]), i.e.

$$
\mathscr{H}_{\alpha}:=\lim _{T \rightarrow \infty} \frac{\mathbb{E}\left(\exp \left(\max _{0 \leq t \leq T} \chi(t)\right)\right)}{T},
$$

where $\chi(t)=\sqrt{2} B_{\alpha / 2}(t)-|t|^{\alpha}$, with $\left\{B_{\alpha / 2}(t): t \geq 0\right\}$ being a fractional Brownian motion with Hurst parameter $\frac{1}{2} \alpha \in(0,1]$. We note in passing that $\mathscr{H}_{\alpha}$ appears for the first time in Pickands' theorem [4]; a correct proof of that theorem was first given by Piterbarg [5].

For an $N(0,1) \mathrm{RV} \mathcal{W}$ we write $\Phi(u)=\mathbb{P}(\mathcal{W} \leq u), \Psi(u)=\mathbb{P}(\mathcal{W}>u)$. Recall that

$$
\Psi(u)=\frac{1}{\sqrt{2 \pi} u} \exp \left(-\frac{u^{2}}{2}\right)(1+o(1)) \quad \text { as } u \rightarrow \infty .
$$

Following Piterbarg [6, Theorem 7.1] we recall that for a centred stationary Gaussian field $\{X(s, t)\}$ satisfying A1 and A2, for arbitrary $g, h \in(0, \infty)$,

$$
\mathbb{P}\left(\max _{(s, t) \in[0, g] \times[0, h]} X(s, t)>u\right)=\mathscr{H}_{\alpha_{1}} \mathscr{H}_{\alpha_{2}} g h u^{2 / \alpha_{1}} u^{2 / \alpha_{2}} \Psi(u)(1+o(1)) \quad \text { as } u \rightarrow \infty .
$$


Let $m_{1}(u) \rightarrow \infty$ and $m_{2}(u) \rightarrow \infty$ be functions such that

$$
m_{1}(u)=\frac{a_{1}(u)}{\sqrt{\Psi(u)}} \quad \text { and } \quad m_{2}(u)=\frac{a_{2}(u)}{\sqrt{\Psi(u)}}
$$

for some positive function $a_{1}(u), a_{2}(u)$ satisfying $a_{1}(u) a_{2}(u)=\left(\mathscr{H}_{\alpha_{1}} \mathcal{H}_{\alpha_{2}} u^{2 / \alpha_{1}} u^{2 / \alpha_{2}}\right)^{-1}$, $\log a_{1}(u)=o\left(u^{2}\right)$ and $\log a_{2}(u)=o\left(u^{2}\right)$. We note that

$$
m(u):=m_{1}(u) m_{2}(u)=\mathbb{P}\left(\max _{(s, t) \in[0,1]^{2}} X(s, t)>u\right)^{-1}(1+o(1)) \quad \text { as } u \rightarrow \infty .
$$

By $\mathscr{B}(0, x)$ we denote a ball in $\mathbb{R}^{2}$ of centre at $(0,0)$ and radius $x$.

\section{Main results}

The aim of this section is to prove a two-dimensional counterpart of Theorem 1. Recall that $\mathcal{W}$ denotes an $N(0,1)$ RV. For a given Jordan-measurable set $\& \subset \mathbb{R}^{2}$ with Lebesgue measure $\operatorname{mes}(\mathcal{E})>0$ let $\mathcal{E}_{u}:=\left\{(x, y):\left(x / m_{1}(u), y / m_{2}(u)\right) \in \mathcal{E}\right\}$. One interesting example is $\varepsilon_{u}=\left[0, x m_{1}(u)\right] \times\left[0, y m_{2}(u)\right]$ for $x, y$ positive, hence, $\mathscr{E}=[0, x] \times[0, y]$ and $\operatorname{mes}(\mathscr{E})=x y$. For such $\varepsilon_{u}$ we shall show (below) an approximation which holds uniformly on compact intervals of $(0, \infty)^{2}$. If the structure of the set is not specified, then for the supremum of a Gaussian field over some general-measurable set $\mathcal{T}_{u} \subset \mathbb{R}^{2}$, an $\epsilon$-net $\left(\mathcal{L}_{\varepsilon}, \mathcal{U}_{\varepsilon}\right)$ approximation of $\mathcal{T}_{u}$ will be assumed. Specifically, the $\epsilon$-net $\left(\mathcal{L}_{\varepsilon}, U_{\varepsilon}\right)$ here means that for any $\varepsilon>0$ there exist two sets $\mathcal{L}_{\varepsilon}$ and $U_{\varepsilon}$ which are simple sets (i.e. finite sums of disjoint rectangles of the form $\left.\left[a_{1}, b_{1}\right) \times\left[a_{2}, b_{2}\right)\right)$ such that

$$
\lim _{\varepsilon \downarrow 0} \operatorname{mes}\left(\mathcal{L}_{\varepsilon}\right)=\lim _{\varepsilon \downarrow 0} \operatorname{mes}\left(\mathcal{U}_{\varepsilon}\right)=c \in(0, \infty)
$$

and

$$
\begin{aligned}
\mathscr{L}_{\varepsilon, u} & =\left\{(x, y):\left(\frac{x}{m_{1}(u)}, \frac{y}{m_{2}(u)}\right) \in \mathcal{L}_{\varepsilon}\right\} \subset \mathcal{T}_{u} \subset \mathcal{U}_{\varepsilon, u} \\
& =\left\{(x, y):\left(\frac{x}{m_{1}(u)}, \frac{y}{m_{2}(u)}\right) \in \mathcal{U}_{\varepsilon}\right\} \subset \mathbb{R}^{2} .
\end{aligned}
$$

Next, we formulate our main results for these two cases.

Theorem 2. Let $\{X(s, t): s, t \geq 0\}$ be a centred homogeneous Gaussian field with covariance function that satisfies A1, A2, and A3 with $r \in[0, \infty)$. Then,

(i) for each $0<A<B<\infty$,

$$
\mathbb{P}\left(\sup _{(s, t) \in\left[0, x m_{1}(u)\right] \times\left[0, y m_{2}(u)\right]} X(s, t) \leq u\right) \rightarrow \mathbb{E}(\exp (-x y \exp (-2 r+2 \sqrt{r} \mathcal{W})))
$$

as $u \rightarrow \infty$, uniformly for $(x, y) \in[A, B]^{2}$.

(ii) for $\mathcal{T}_{u} \subset \mathbb{R}^{2}, u>0$ such that there exists an $\epsilon$-net $\left(\mathcal{L}_{\varepsilon}, U_{\varepsilon}\right)$ satisfying (2)

$$
\mathbb{P}\left(\sup _{(s, t) \in \mathcal{T}_{u}} X(s, t) \leq u\right) \rightarrow \mathbb{E}(\exp (-c \exp (-2 r+2 \sqrt{r} W))) \quad \text { as } u \rightarrow \infty .
$$

The complete proof of Theorem 2 is given in Section 5.1. 
Remark 1. Following the same reasoning as given in the proof of Theorem 2, assuming that A1-A3 hold, for each $0<A<B<\infty$, we have

$$
\mathbb{P}\left(\sup _{(s, t) \in \mathcal{B}(0, x \sqrt{m(u)})} X(s, t) \leq u\right) \rightarrow \mathbb{E}\left(\exp \left(-\pi x^{2} \exp (-2 r+2 \sqrt{r} w)\right)\right)
$$

as $u \rightarrow \infty$, uniformly for $x \in[A, B] ; \mathcal{B}(0, x)$ is a ball in $\mathbb{R}^{2}$ of centre at $(0,0)$ and radius $x$.

\section{Applications}

In this section we apply our main results to the analysis of the asymptotic properties of supremum of a Gaussian field over a random parameter set and to the analysis of the dependance structure of homogeneous Gaussian fields.

\subsection{Extremes of homogeneous Gaussian fields over a random parameter set}

In this section we analyse the asymptotic properties of the tail distribution of

$$
\sup _{(s, t) \in \mathscr{B}(0, T)} X(s, t),
$$

where $T$ is a nonnegative, independent of the $X \mathrm{RV}$. The one-dimensional counterpart of this problem was recently analysed in [2] and [3].

Proposition 1. Let $\{X(s, t): s, t \geq 0\}$ be a centred homogeneous Gaussian field with covariance function that satisfies A1-A3 with $r \in[0, \infty)$, and let $T$ be an independent of the $X$ nonnegative $R V$.

(i) If $\mathbb{E}\left(T^{2}\right)<\infty$, then, as $u \rightarrow \infty$,

$$
\mathbb{P}\left(\sup _{(s, t) \in \mathscr{B}(0, T)} X(s, t)>u\right)=\pi \mathbb{E}\left(T^{2}\right) \mathscr{H}_{\alpha_{1}} \mathcal{H}_{\alpha_{2}} u^{2 / \alpha_{1}} u^{2 / \alpha_{2}} \Psi(u)(1+o(1)) .
$$

(ii) If $T$ has a regularly varying tail distribution at $\infty$ with index $\lambda \in(0,2)$, then, as $u \rightarrow \infty$,

$$
\mathbb{P}\left(\sup _{(s, t) \in \mathscr{B}(0, T)} X(s, t)>u\right)=2 \pi K \mathbb{P}(T>\sqrt{m(u)})(1+o(1)),
$$

where $K=\int_{0}^{\infty} x^{1-\lambda} \mathbb{E}\left(\exp \left(-\pi x^{2} \boldsymbol{W}_{r}+\log \boldsymbol{W}_{r}\right)\right) \mathrm{d} x$ and $\boldsymbol{W}_{r}=\exp (2 \sqrt{r} \boldsymbol{W}-2 r)$.

(iii) If $T$ has a slowly varying tail distribution at $\infty$, then, as $u \rightarrow \infty$,

$$
\mathbb{P}\left(\sup _{(s, t) \in \mathscr{B}(0, T)} X(s, t)>u\right)=\mathbb{P}(T>\sqrt{m(u)})(1+o(1)) .
$$

The proof of Proposition 1 is given in Section 5.2; for details on regularly varying functions see the classical monographs [7] and [8].

\subsection{Extremal indices for homogeneous Gaussian fields}

Following [9], we say that $\theta \in(0,1]$ is the extremal index of a homogeneous discreteparameter stationary random field $\left\{X_{j, k}: j, k=1,2, \ldots\right\}$, if

$$
\mathbb{P}\left(\max _{j \leq a_{n}, k \leq b_{n}} X_{j, k} \leq z_{n}\right)-\mathbb{P}\left(X_{1,1} \leq z_{n}\right)^{a_{n} b_{n} \cdot \theta} \rightarrow 0,
$$

as $n \rightarrow \infty$, for each sequence $\left(z_{n}\right) \subset \mathbb{R}$ and all sequences $\left(a_{n}\right),\left(b_{n}\right) \subset \mathbb{N}$ such that $a_{n} \rightarrow \infty$ and $b_{n} \rightarrow \infty$, as $n \rightarrow \infty$, and $1 / C \leq a_{n} / b_{n} \leq C$ for some fixed arbitrary constant $C>0$. 
The notion of extremal index $\theta$ originated in the investigations concerning the relationship between the dependence structure of discrete-parameter stationary sequences of RVs and their extremal behaviour [1], [10]; see also [11]-[15]. For a given centred homogeneous Gaussian field $\{X(s, t): s, t \geq 0\}$ that satisfies A1-A3 introduce a discrete-parameter random field $\left\{\widetilde{X}_{j, k}: j, k=1,2, \ldots\right\}$, with

$$
\widetilde{X}_{j, k}:=\sup _{(s, t) \in[j-1, j] \times[k-1, k]} X(s, t) .
$$

In the following proposition we point out how the difference in the dependance structure between weakly and strongly dependant Gaussian fields influences the existence of the extremal index of the associated field $\left\{\widetilde{X}_{j, k}\right\}$.

Proposition 2. We assume that A1-A3 hold for a centred homogeneous Gaussian field where $\{X(s, t): s, t \geq 0\}$.

(i) If $r=0$ then the extremal index of $\left\{\widetilde{X}_{j, k}: j, k=1,2, \ldots\right\}$ equals to 1.

(ii) If $r>0$ then $\left\{\tilde{X}_{j, k}: j, k=1,2, \ldots\right\}$ does not have an extremal index.

The proof of Proposition 2 is deferred to Section 5.3.

\section{Proofs}

Before we prove Theorem 2, we need some auxiliary results. Lemma 1 is a two-dimensional version of Lemma 12.2.11 of [1]. Lemma 2 combines a two-dimensional counterpart of Lemma 12.3.1 of [1] for weakly dependent fields, and Lemma 3.1 of [3] for strongly dependent fields. We omit the proofs of the first three lemmas, which are given in the full-length version of this paper [17].

Lemma 1. Assume that $A 1$ and $A 2$ hold, and $q_{1}=q_{1}(u)=a u^{-2 / \alpha_{1}}$ and $q_{2}=q_{2}(u)=$ $a u^{-2 / \alpha_{2}}$ for some $a>0$. Then, for any $x, y \geq 0, g, h>0$ and rectangle $I=(x, y)+[0, g] \times$ $[0, h]$, as $u \rightarrow \infty$,

$$
\mathbb{P}\left(X\left(j q_{1}, k q_{2}\right) \leq u ;\left(j q_{1}, k q_{2}\right) \in I\right)-\mathbb{P}(X(s, t) \leq u ;(s, t) \in I) \leq \frac{g h \rho(a)}{m(u)}+o\left(\frac{1}{m(u)}\right),
$$

where $\rho(a) \rightarrow 0$ as $a \rightarrow 0$.

Next, let

$$
\begin{gathered}
\rho_{T}(s, t):= \begin{cases}1, & 0 \leq \max (|s|,|t|)<1, \\
\left|r(s, t)-\frac{r}{\log T}\right|, & 1 \leq \max (|s|,|t|) \leq T,\end{cases} \\
\varrho_{T}(s, t):= \begin{cases}|r(s, t)|+(1-r(s, t)) \frac{r}{\log T}, & 0 \leq \max (|s|,|t|)<1, \\
\frac{r}{\log T}, & 1 \leq \max (|s|,|t|) \leq T .\end{cases}
\end{gathered}
$$


Lemma 2. Let $\varepsilon>0$ be given. Let $q_{1}=q_{1}(u)=a u^{-2 / \alpha_{1}}$ and $q_{2}=q_{2}(u)=a u^{-2 / \alpha_{2}}$. Suppose that $T_{1}=T_{1}(u) \sim \tau m_{1}(u)$ and $T_{2}=T_{2}(u) \sim \tau m_{2}(u)$ for some $\tau>0$, as $u \rightarrow \infty$. Then, providing that conditions $A 1, A 2$, and $A 3$ with $r \in[0, \infty)$ are fulfilled,

$$
\frac{T_{1} T_{2}}{q_{1} q_{2}} \sum_{\mathcal{C}} \rho_{T_{\max }}\left(j q_{1}, k q_{2}\right) \exp \left(\frac{-u^{2}}{1+\max \left(\left|r\left(j q_{1}, k q_{2}\right)\right|, \varrho_{T_{\max }}\left(j q_{1}, k q_{2}\right)\right)}\right) \rightarrow 0
$$

as $u \rightarrow \infty$, where $T_{\max }=\max \left(T_{1}, T_{2}\right)$ and $\mathcal{C}=\left\{\left(j q_{1}, k q_{2}\right) \in\left[-T_{1}, T_{1}\right] \times\left[-T_{2}, T_{2}\right]-\right.$ $\left.(-\varepsilon, \varepsilon)^{2}\right\}$ in the summation.

Lemma 3. Let $_{1}=q_{1}(u)=a u^{-2 / \alpha_{1}}, q_{2}=q_{2}(u)=a u^{-2 / \alpha_{2}}$, and suppose that $T=T(u) \rightarrow$ $\infty$, as $u \rightarrow \infty$. Then, providing that conditions $A 1$ and $A 2$ are fulfilled, there exists $\varepsilon>0$ such that

$$
\begin{aligned}
\frac{m(u)}{q_{1} q_{2}} \sum_{0<\max \left(\left|j q_{1}\right|,\left|k q_{2}\right|\right)<\varepsilon} & {\left[\left(1-r\left(j q_{1}, k q_{2}\right)\right) \frac{r}{\log T}\right.} \\
& \times\left(1-\left(r\left(j q_{1}, k q_{2}\right)+\left(1-r\left(j q_{1}, k q_{2}\right)\right) \frac{r}{\log T}\right)^{2}\right)^{-1 / 2} \\
& \left.\times \exp \left(-\frac{u^{2}}{1+r\left(j q_{1}, k q_{2}\right)+\left(1-r\left(j q_{1}, k q_{2}\right)\right)(r / \log T)}\right)\right] \rightarrow 0
\end{aligned}
$$

as $u \rightarrow \infty$.

\subsection{Proof of Theorem 2}

Proof of $(i)$. Let $\left\{X^{(j, k)}(s, t)\right\}_{j, k}$ be independent copies of $X(s, t)$, and let $\eta(s, t)$ be such that $\eta(s, t)=X^{(j, k)}(s, t)$ for $(s, t) \in[j-1, j) \times[k-1, k)$. For a fixed $T$ we define a Gaussian random field $Y_{T}$ as follows:

$$
Y_{T}(s, t):=\left(1-\frac{r}{\log T}\right)^{1 / 2} \eta(s, t)+\left(\frac{r}{\log T}\right)^{1 / 2} \mathcal{W} \quad \text { for }(s, t) \in[0, T]^{2},
$$

where $\mathcal{W}$ is an $N(0,1) \mathrm{RV}$ independent of $\eta(s, t)$. Then the covariance of $Y_{T}$ equals

$$
\begin{aligned}
& \operatorname{cov}\left(Y_{T}\left(s_{0}, t_{0}\right), Y_{T}\left(s_{0}+s, t_{0}+t\right)\right) \\
& = \begin{cases}r(s, t)+(1-r(s, t)) \frac{r}{\log T} & \text { when }\left[s_{0}\right]=\left[s_{0}+s\right],\left[t_{0}\right]=\left[t_{0}+t\right], \\
\frac{r}{\log T} & \text { otherwise, }\end{cases}
\end{aligned}
$$

for all $s_{0}, t_{0}, s, t \geq 0$.

Let $n_{x}:=\left\lfloor x m_{1}(u)\right\rfloor$ and $n_{y}:=\left\lfloor y m_{2}(u)\right\rfloor$. Since

$$
\begin{aligned}
\mathbb{P}\left(\sup _{(s, t) \in\left[0, n_{x}+1\right] \times\left[0, n_{y}+1\right]} X(s, t) \leq u\right) & \leq \mathbb{P}\left(\sup _{(s, t) \in\left[0, x m_{1}(u)\right] \times\left[0, y m_{2}(u)\right]} X(s, t) \leq u\right) \\
& \leq \mathbb{P}\left(\sup _{(s, t) \in\left[0, n_{x}\right] \times\left[0, n_{y}\right]} X(s, t) \leq u\right),
\end{aligned}
$$

we focus on the asymptotics of $\mathbb{P}\left(\sup _{(s, t) \in\left[0, n_{x}\right] \times\left[0, n_{y}\right]} X(s, t) \leq u\right)$, as $u \rightarrow \infty$. Let $\varepsilon>0$. 
Divide $\left[0, n_{x}\right] \times\left[0, n_{y}\right]$ into $n_{x} n_{y}$ unit squares and then split them into subsets $I_{l, m}^{*}$ and $I_{l, m}$ as follows,

$$
I_{l, m}=[(l-1)+\varepsilon, l] \times[(m-1)+\varepsilon, m], \quad I_{l, m}^{*}=[l-1, l] \times[m-1, m]-I_{l, m},
$$

where $l=1, \ldots, n_{x}, m=1, \ldots, n_{y}$.

Step 1. We prove that

$$
\lim _{u \rightarrow \infty}\left|\mathbb{P}\left(\sup _{(s, t) \in\left[0, n_{x}\right] \times\left[0, n_{y}\right]} X(s, t) \leq u\right)-\mathbb{P}\left(\sup _{(s, t) \in \bigcup_{l=1}^{n_{x}} \bigcup_{m=1}^{n_{y}} I_{l, m}} X(s, t) \leq u\right)\right| \leq \rho_{1}(\varepsilon),
$$

uniformly for $(x, y) \in[A, B]^{2}$ with $\rho_{1}(\varepsilon) \rightarrow 0$ as $\varepsilon \rightarrow 0$. This is a consequence of the following sequence of inequalities:

$$
\begin{aligned}
0 & \leq \mathbb{P}\left(\sup _{(s, t) \in \bigcup_{l=1}^{n_{x}} \bigcup_{m=1}^{n_{y}} I_{l, m}} X(s, t) \leq u\right)-\mathbb{P}\left(\sup _{(s, t) \in\left[0, n_{x}\right] \times\left[0, n_{y}\right]} X(s, t) \leq u\right) \\
& \leq n_{x} n_{y} \mathbb{P}\left(\sup _{(s, t) \in I_{1,1}^{*}} X(s, t)>u\right) \\
& \leq B^{2} m(u) \mathbb{P}\left(\sup _{(s, t) \in I_{1,1}^{*}} X(s, t)>u\right) \\
& =\left(2 \varepsilon-\varepsilon^{2}\right) B^{2}(1+o(1)),
\end{aligned}
$$

as $u \rightarrow \infty$, since

$$
\mathbb{P}\left(\sup _{(s, t) \in I_{1,1}^{*}} X(s, t)>u\right)=\frac{2 \varepsilon-\varepsilon^{2}}{m(u)}(1+o(1)),
$$

as $u \rightarrow \infty$, by [6, Theorem 7.1].

Step 2. Let $a>0, q_{1}=q_{1}(u):=a u^{-\alpha_{1} / 2}$, and $q_{2}=q_{2}(u):=a u^{-\alpha_{2} / 2}$. We show that

$$
\begin{aligned}
& \lim _{u \rightarrow \infty} \mid \mathbb{P}\left(X(s, t) \leq u ;(s, t) \in \bigcup_{l=1}^{n_{x}} \bigcup_{m=1}^{n_{y}} I_{l, m}\right) \\
& \quad-\mathbb{P}\left(X\left(j q_{1}, k q_{2}\right) \leq u ;\left(j q_{1}, k q_{2}\right) \in \bigcup_{l=1}^{n_{x}} \bigcup_{m=1}^{n_{y}} I_{l, m}\right) \mid \\
& \leq \rho_{2}(a),
\end{aligned}
$$

uniformly for $(x, y) \in[A, B]^{2}$, with $\rho_{2}(a) \rightarrow 0$ as $a \rightarrow 0$. Indeed, (5) follows from the fact that

$$
\begin{aligned}
0 \leq & \mathbb{P}\left(X(s, t) \leq u ;(s, t) \in \bigcup_{l=1}^{n_{x}} \bigcup_{m=1}^{n_{y}} I_{l, m}\right) \\
& -\mathbb{P}\left(X\left(j q_{1}, k q_{2}\right) \leq u ;\left(j q_{1}, k q_{2}\right) \in \bigcup_{l=1}^{n_{x}} \bigcup_{m=1}^{n_{y}} I_{l, m}\right) \\
\leq & n_{x} n_{y} \max _{l, m}\left[\mathbb{P}\left(X\left(j q_{1}, k q_{2}\right) \leq u ;\left(j q_{1}, k q_{2}\right) \in I_{l, m}\right)-\mathbb{P}\left(\sup _{(s, t) \in I_{l, m}} X(s, t) \leq u\right)\right]
\end{aligned}
$$




$$
\begin{aligned}
& \leq n_{x} n_{y}(1-\varepsilon)^{2}\left(\frac{\rho(a)}{m(u)}+o\left(\frac{1}{m(u)}\right)\right) \\
& \leq B^{2} \rho(a)+B^{2} m(u) o\left(\frac{1}{m(u)}\right) \\
& \rightarrow B^{2} \rho(a)
\end{aligned}
$$

as $u \rightarrow \infty$, with $\rho(a) \rightarrow 0$ as $a \rightarrow 0$. Inequality (6) is due to Lemma 1 .

Step 3. We show that for $T=T(u):=\max \left(A_{\infty} m_{1}(u), A_{\infty} m_{2}(u)\right)$ we have

$$
\begin{aligned}
& \mid \mathbb{P}\left(X\left(j q_{1}, k q_{2}\right) \leq u ;\left(j q_{1}, k q_{2}\right) \in \bigcup_{l=1}^{n_{x}} \bigcup_{m=1}^{n_{y}} I_{l, m}\right) \\
& \quad-\mathbb{P}\left(Y_{T}\left(j q_{1}, k q_{2}\right) \leq u ;\left(j q_{1}, k q_{2}\right) \in \bigcup_{l=1}^{n_{x}} \bigcup_{m=1}^{n_{y}} I_{l, m}\right) \mid \\
& \rightarrow 0,
\end{aligned}
$$

as $u \rightarrow \infty$, uniformly for $(x, y) \in[A, B]^{2}$. For sufficiently large $T$ we have

$$
\begin{aligned}
& \left|\operatorname{cov}\left(X\left(j q_{1}, k q_{2}\right), X\left(j^{\prime} q_{1}, k^{\prime} q_{2}\right)\right)-\operatorname{cov}\left(Y_{T}\left(j q_{1}, k q_{2}\right), Y_{T}\left(j^{\prime} q_{1}, k^{\prime} q_{2}\right)\right)\right| \\
& \quad \leq \rho_{T}\left(\left(j-j^{\prime}\right) q_{1},\left(k-k^{\prime}\right) q_{2}\right)
\end{aligned}
$$

and

$$
\left|\operatorname{cov}\left(Y_{T}\left(j q_{1}, k q_{2}\right), Y_{T}\left(j^{\prime} q_{1}, k^{\prime} q_{2}\right)\right)\right| \leq \varrho_{T}\left(\left(j-j^{\prime}\right) q_{1},\left(k-k^{\prime}\right) q_{2}\right),
$$

for functions $\rho_{T}$ and $\varrho_{T}$ defined by (5). Moreover, for small $\varepsilon>0$ and $\left(j q_{1}, k q_{2}\right),\left(j^{\prime} q_{1}, k^{\prime} q_{2}\right) \in$ $\cup_{l=1}^{n_{x}} \cup_{m=1}^{n_{y}} I_{l, m}$ satisfying $\max \left(\left|j-j^{\prime}\right| q_{1},\left|k-k^{\prime}\right| q_{2}\right)<\varepsilon$ we obtain

$$
\begin{aligned}
& \left|\operatorname{cov}\left(X\left(j q_{1}, k q_{2}\right), X\left(j^{\prime} q_{1}, k^{\prime} q_{2}\right)\right)-\operatorname{cov}\left(Y_{T}\left(j q_{1}, k q_{2}\right), Y_{T}\left(j^{\prime} q_{1}, k^{\prime} q_{2}\right)\right)\right| \\
& =\left(1-r\left(\left(j-j^{\prime}\right) q_{1},\left(k-k^{\prime}\right) q_{2}\right)\right) \frac{r}{\log T}
\end{aligned}
$$

and

$\max \left(\left|\operatorname{cov}\left(X\left(j q_{1}, k q_{2}\right), X\left(j^{\prime} q_{1}, k^{\prime} q_{2}\right)\right)\right|, \mid \operatorname{cov}\left(Y_{T}\left(j q_{1}, k q_{2}\right), Y_{T}\left(j^{\prime} q_{1}, k^{\prime} q_{2}\right) \mid\right)\right.$

$$
\begin{aligned}
& =\operatorname{cov}\left(Y_{T}\left(j q_{1}, k q_{2}\right), Y_{T}\left(j^{\prime} q_{1}, k^{\prime} q_{2}\right)\right) \\
& =r\left(\left(j-j^{\prime}\right) q_{1},\left(k-k^{\prime}\right) q_{2}\right)+\left(1-r\left(\left(j-j^{\prime}\right) q_{1},\left(k-k^{\prime}\right) q_{2}\right)\right) \frac{r}{\log T} .
\end{aligned}
$$

Let $\delta_{T}=\sup \left\{\max \left(|r(s, t)|, \varrho_{T}(s, t)\right) ; \max (|s|,|t|) \geq \varepsilon\right.$. Observe that $\delta_{T}<\delta<1$ for sufficiently large $T$. Applying [1, Theorem 4.2.1] we obtain

$$
\begin{aligned}
& \mid \mathbb{P}\left(X\left(j q_{1}, k q_{2}\right) \leq u ;\left(j q_{1}, k q_{2}\right) \in \bigcup_{l=1}^{n_{x}} \bigcup_{m=1}^{n_{y}} I_{l, m}\right) \\
& \quad-\mathbb{P}\left(Y_{T}\left(j q_{1}, k q_{2}\right) \leq u ;\left(j q_{1}, k q_{2}\right) \in \bigcup_{l, m} I_{l, m}\right) \mid
\end{aligned}
$$




$$
\begin{aligned}
& \leq \frac{1}{4 \pi} \frac{n_{x} n_{y}}{q_{1} q_{2}} \sum_{D}\left[\left(1-r\left(j q_{1}, k q_{2}\right)\right) \frac{r}{\log T}\right. \\
& \times\left(1-\left(r\left(j q_{1}, k q_{2}\right)+\left(1-r\left(j q_{1}, k q_{2}\right)\right) \frac{r}{\log T}\right)^{2}\right)^{-1 / 2} \\
& \left.\times \exp \left(-\frac{u^{2}}{1+r\left(j q_{1}, k q_{2}\right)+\left(1-r\left(j q_{1}, k q_{2}\right)\right)(r / \log T)}\right)\right] \\
& +\frac{1}{4 \pi}\left(1-\delta^{2}\right)^{-1 / 2} \frac{n_{x} n_{y}}{q_{1} q_{2}} \sum_{\mathcal{E}}\left[\rho_{T}\left(j q_{1}, k q_{2}\right) \mathrm{e}^{-u^{2} /\left(1+\max \left(\left|r\left(j q_{1}, k q_{2}\right)\right|, \varrho_{T}\left(j q_{1}, k q_{2}\right)\right)\right.}\right] \\
& \leq \frac{1}{4 \pi} \frac{B^{2} m(u)}{q_{1} q_{2}} \sum_{\mathcal{F}}\left[\left(1-r\left(j q_{1}, k q_{2}\right)\right) \frac{r}{\log T}\right. \\
& \times\left(1-\left(r\left(j q_{1}, k q_{2}\right)+\left(1-r\left(j q_{1}, k q_{2}\right)\right) \frac{r}{\log T}\right)^{2}\right)^{-1 / 2} \\
& \left.\times \exp \left(-\frac{u^{2}}{1+r\left(j q_{1}, k q_{2}\right)+\left(1-r\left(j q_{1}, k q_{2}\right)\right)(r / \log T)}\right)\right] \\
& +\frac{1}{4 \pi}\left(1-\delta^{2}\right)^{-1 / 2} \frac{B^{2} m(u)}{q_{1} q_{2}} \\
& \times \sum_{g}\left[\rho_{T}\left(j q_{1}, k q_{2}\right) \exp \left(-\frac{u^{2}}{1+\max \left(\left|r\left(j q_{1}, k q_{2}\right)\right|, \varrho_{T}\left(j q_{1}, k q_{2}\right)\right)}\right)\right] \\
& =: I_{1}+I_{2} \text {, }
\end{aligned}
$$

where $\mathscr{D}=\left\{0<\max \left(\left|j q_{1}\right|,\left|k q_{2}\right|\right)<\varepsilon\right\}, \mathcal{E}=\left\{\left(j q_{1}, k q_{2}\right) \in\left[-n_{x}, n_{x}\right] \times\left[-n_{y}, n_{y}\right]-\right.$ $\left.(-\varepsilon, \varepsilon)^{2}\right\}, \mathcal{F}=\left\{0<\max \left(\left|j q_{1}\right|,\left|k q_{2}\right|\right)<\varepsilon\right\}$, and $\mathcal{G}=\left\{\left(j q_{1}, k q_{2}\right) \in\left[-B m_{1}(u), B m_{1}(u)\right] \times\right.$ $\left.\left[-B m_{2}(u), B m_{2}(u)\right]-(-\varepsilon, \varepsilon)^{2}\right\}$ in the summations. By Lemma $3, I_{1}$ tends to 0 as $u \rightarrow \infty$. Analogously, by Lemma 2, $I_{2}$ tends to 0 as $u \rightarrow \infty$. Hence, we have shown (7).

Step 4. By the definition of the random field $Y_{T}$, we have

$$
\begin{aligned}
\mathbb{P}\left(Y_{T}\left(j q_{1}, k q_{2}\right) \leq u ;\left(j q_{1}, k q_{2}\right) \in \bigcup_{l, m} I_{l, m}\right) \\
\quad=\mathbb{P}\left(\left(1-\frac{r}{\log T}\right)^{1 / 2} \eta\left(j q_{1}, k q_{2}\right)+\left(\frac{r}{\log T}\right)^{1 / 2} \mathcal{W} \leq u ;\left(j q_{1}, k q_{2}\right) \in \bigcup_{l, m} I_{l, m}\right) \\
\quad=\mathbb{P}\left(\left(1-\frac{r}{\log T}\right)^{1 / 2} \sup _{\left(j q_{1}, k q_{2}\right) \in \bigcup_{l, m} I_{l, m}} \eta\left(j q_{1}, k q_{2}\right)+\left(\frac{r}{\log T}\right)^{1 / 2} \mathcal{W} \leq u\right) \\
\quad=\int_{-\infty}^{\infty} \mathbb{P}\left(\sup _{\left(j q_{1}, k q_{2}\right) \in \bigcup_{l, m} I_{l, m}} \eta\left(j q_{1}, k q_{2}\right) \leq \frac{u-(r / \log T)^{1 / 2} z}{(1-r / \log T)^{1 / 2}}\right) \mathrm{d} \Phi(z) .
\end{aligned}
$$

Then, for any $z \in \mathbb{R}$,

$$
u_{z}:=\frac{u-(r / \log T)^{1 / 2} z}{(1-r / \log T)^{1 / 2}}=u+\frac{-2 \sqrt{r} z+2 r}{u}+o\left(\frac{1}{u}\right) \quad \text { as } u \rightarrow \infty,
$$


and, thus,

$$
\frac{1}{m\left(u_{z}\right)}=\frac{\exp (-2 r+2 \sqrt{r} z)}{m(u)}(1+o(1))
$$

Hence, we obtain

$$
\begin{aligned}
\mathbb{P}\left(\sup _{\left(j q_{1}, k q_{2}\right) \in \bigcup_{l, m} I_{l, m}} \eta\left(j q_{1}, k q_{2}\right) \leq u_{z}\right) & =\prod_{l, m} \mathbb{P}\left(\sup _{\left(j q_{1}, k q_{2}\right) \in I_{l, m}} X\left(j q_{1}, k q_{2}\right) \leq u_{z}\right) \\
& =\mathbb{P}\left(\sup _{(s, t) \in[0,1]^{2}} X(s, t) \leq u_{z}\right)^{n_{x} n_{y}}(1+o(1)) \\
& =\left(1-\frac{1}{m\left(u_{z}\right)}\right)^{x y m(u)}(1+o(1)) \\
& =\exp (-x y \exp (-2 r+2 \sqrt{r} z))(1+o(1))
\end{aligned}
$$

as $u \rightarrow \infty$, uniformly for $(x, y) \in\left[A, A_{\infty}\right]^{2}$. Combining (4), (5), (7), (8), and (9), and passing with $\varepsilon \rightarrow 0$ and $a \rightarrow 0$, we conclude that the proof of (i) is complete.

Proof of (ii). Following line by line the same argument as given in the proof of part (i) of Theorem 2, the assumption of the existence of the $\epsilon$-net $\left(\mathcal{L}_{\varepsilon}, U_{\varepsilon}\right)$ implies that

$$
\mathbb{P}\left(\sup _{(s, t) \in \mathscr{L}_{\varepsilon, u}} X(s, t) \leq u\right) \rightarrow \mathbb{E}\left(\exp \left(-\operatorname{mes}\left(\mathcal{L}_{\varepsilon}\right) \exp (-2 r+2 \sqrt{r} \mathcal{W})\right)\right)
$$

and

$$
\mathbb{P}\left(\sup _{(s, t) \in \mathcal{U}_{\varepsilon, u}} X(s, t) \leq u\right) \rightarrow \mathbb{E}\left(\exp \left(-\operatorname{mes}\left(\mathcal{U}_{\varepsilon}\right) \exp (-2 r+2 \sqrt{r} w)\right)\right),
$$

as $u \rightarrow \infty$. Thus,

$$
\mathbb{P}\left(\sup _{(s, t) \in \mathcal{T}_{u}} X(s, t) \leq u\right) \rightarrow \mathbb{E}(\exp (-c \exp (-2 r+2 \sqrt{r} \mathcal{W})))
$$

as $u \rightarrow \infty$.

\subsection{Proof of Proposition 1}

Since the proof of Proposition 1 is analogous to the proofs of Theorems 3.1-3.3 of [2], see also Theorem A of [3], we focus only on the arguments for (ii).

Let $0<A<B$. We have

$$
\begin{aligned}
\mathbb{P}\left(\sup _{(s, t) \in \mathcal{B}(0, T)} X(s, t)>u\right)= & \int_{0}^{A \sqrt{m(u)}} \mathbb{P}\left(\sup _{(s, t) \in \mathcal{B}(0, x)} X(s, t)>u\right) \mathrm{d} F_{T}(x) \\
& +\int_{A \sqrt{m(u)}}^{B \sqrt{m(u)}} \mathbb{P}\left(\sup _{(s, t) \in \mathcal{B}(0, x)} X(s, t)>u\right) \mathrm{d} F_{T}(x) \\
& +\int_{B \sqrt{m(u)}}^{\infty} \mathbb{P}\left(\sup _{(s, t) \in \mathcal{B}(0, x)} X(s, t)>u\right) \mathrm{d} F_{T}(x) \\
= & I_{1}+I_{2}+I_{3} .
\end{aligned}
$$


Then, for each $\varepsilon>0$, due to Remark 1 , for sufficiently large $u$, with $\mathcal{W}_{r}=\exp (2 \sqrt{r} \mathcal{W}-2 r)$, we obtain

$$
\begin{aligned}
I_{2} \leq & (1+\varepsilon) \int_{A}^{B}\left(1-\mathbb{E}\left(\exp \left(-\pi x^{2} \mathcal{W}_{r}\right)\right) \mathrm{d} F_{T}(x \sqrt{m(u)})\right. \\
= & (1+\varepsilon) \int_{A}^{B} 2 \pi x \mathbb{E}\left(\exp \left(-\pi x^{2} \mathcal{W}_{r}+\log \mathcal{W}_{r}\right)\right) \mathbb{P}(T>x \sqrt{m(u)}) \mathrm{d} x \\
& -(1+\varepsilon)\left(1-\mathbb{E}\left(\exp \left(-\pi B^{2} \mathcal{W}_{r}\right)\right)\right) \mathbb{P}(T>B \sqrt{m(u)}) \\
& +(1+\varepsilon)\left(1-\mathbb{E}\left(\exp \left(-\pi A^{2} W_{r}\right)\right)\right) \mathbb{P}(T>A \sqrt{m(u)}) .
\end{aligned}
$$

Hence, using the fact that $T$ has a regularly varying tail distribution,

$$
\begin{aligned}
\limsup _{u \rightarrow \infty} \frac{I_{2}}{\mathbb{P}(T>\sqrt{m(u)})} \leq & (1+\varepsilon) 2 \pi \int_{A}^{B} x^{1-\lambda} \mathbb{E}\left(\exp \left(-\pi x^{2} \mathcal{W}_{r}+\log \mathcal{W}_{r}\right)\right) \mathrm{d} x \\
& -(1+\varepsilon)\left(1-\mathbb{E}\left(\exp \left(-\pi B^{2} \boldsymbol{W}_{r}\right)\right)\right) B^{-\lambda} \\
& +(1+\varepsilon)\left(1-\mathbb{E}\left(\exp \left(-\pi A^{2} \mathcal{W}_{r}\right)\right)\right) A^{-\lambda} .
\end{aligned}
$$

In an analogous way it follows that

$$
\begin{aligned}
\liminf _{u \rightarrow \infty} \frac{I_{2}}{\mathbb{P}(T>\sqrt{m(u)})} \geq & (1-\varepsilon) 2 \pi \int_{A}^{B} x^{1-\lambda} \mathbb{E}\left(\exp \left(-\pi x^{2} \mathcal{W}_{r}+\log \mathcal{W}_{r}\right)\right) \mathrm{d} x \\
& -(1-\varepsilon)\left(1-\mathbb{E}\left(\exp \left(-\pi B^{2} \mathcal{W}_{r}\right)\right)\right) B^{-\lambda} \\
& +(1-\varepsilon)\left(1-\mathbb{E}\left(\exp \left(-\pi A^{2} \mathcal{W}_{r}\right)\right)\right) A^{-\lambda} .
\end{aligned}
$$

Then, following the same argument as in the proof of Theorem 3.2 of [2], we conclude that $I_{1}+I_{3}=o(\mathbb{P}(T>\sqrt{m(u)}))$ as $u \rightarrow \infty$. Now, passing with $A \rightarrow 0, B \rightarrow \infty$, and $\varepsilon \rightarrow 0$ yields

$I_{2}=2 \pi \int_{0}^{\infty} x^{1-\lambda} \mathbb{E}\left(\exp \left(-\pi x^{2} \mathcal{W}_{r}+\log \mathcal{W}_{r}\right)\right) \mathrm{d} x \mathbb{P}(T>\sqrt{m(u)})(1+o(1)), \quad \geq u \rightarrow \infty$.

\subsection{Proof of Proposition 2}

Proof of $(i)$. Assume that A3 is satisfied with $r=0$. Then, by the definition of $\left\{\widetilde{X}_{j, k}\right\}$, it suffices to show that for the original Gaussian field $\{X(s, t): s, t \geq 0\}$,

$$
\mathbb{P}\left(\sup _{(s, t) \in[0, f(u)] \times[0, g(u)]} X(s, t) \leq z(u)\right)-\mathbb{P}\left(\sup _{(s, t) \in[0,1]^{2}} X(s, t) \leq z(u)\right)^{f(u) g(u)} \rightarrow 0,
$$

as $u \rightarrow \infty$, for each function $z: \mathbb{R}_{+} \rightarrow \mathbb{R}$ and all pairs of functions $f, g: \mathbb{R}_{+} \rightarrow \mathbb{R}_{+}$such that $f(u) \rightarrow \infty$ and $g(u) \rightarrow \infty$, as $u \rightarrow \infty$, and $1 / C \leq f(u) / g(u) \leq C$ for some fixed arbitrary constant $C>0$. Observe that it suffices to consider two cases: continuous $z(u) \nearrow \infty$, as $u \rightarrow \infty$, and $z(u)<C<\infty$. We focus on the first case and suppose that $z(u)$ increases to infinity. Then (10) is equivalent to

$$
\mathbb{P}\left(\sup _{(s, t) \in\left[0, f^{*}(u)\right] \times\left[0, g^{*}(u)\right]} X(s, t) \leq u\right)-\mathbb{P}\left(\sup _{(s, t) \in[0,1]^{2}} X(s, t) \leq u\right)^{f^{*}(u) g^{*}(u)} \rightarrow 0,
$$

as $u \rightarrow \infty$, with $z^{-1}$ being the inverse function for $z$ and $f^{*}(u):=f\left(z^{-1}(u)\right), g^{*}(u):=$ $g\left(z^{-1}(u)\right)$. 
By (i) of Theorem 2,

$$
\mathbb{P}\left(\sup _{(s, t) \in[0, x \sqrt{m(u)}] \times[0, y \sqrt{m(u)}]} X(s, t) \leq u\right) \rightarrow \mathrm{e}^{-x y},
$$

as $u \rightarrow \infty$, uniformly for $(x, y) \in \mathcal{F}(C):=\left\{(s, t) \in \mathbb{R}_{+}^{2}: 1 / C \leq s / t \leq C\right\} \cup\{0,0\}$, for $C>0$.

Moreover, the uniform convergence

$$
\mathbb{P}\left(\sup _{(s, t) \in[0,1]^{2}} X(s, t) \leq u\right)^{x y \cdot m(u)} \rightarrow \mathrm{e}^{-x y}
$$

occurs on the set $\mathcal{F}(C)$.

Let $\bar{f}(u):=f\left(z^{-1}(u)\right) / \sqrt{m(u)}$ and $\bar{g}(u):=g\left(z^{-1}(u)\right) / \sqrt{m(u)}$. The fundamental observation is that it is sufficient to prove (10) for $f(u)$ and $g(u)$ satisfying the additional assumption: $\bar{f}(u) \rightarrow a \in[0, \infty]$ and $\bar{g}(u) \rightarrow b \in[0, \infty]$, as $u \rightarrow \infty$.

Note that $1 / C \leq f(u) / g(u) \leq C$ implies that $1 / C \leq \bar{f}(u) / \bar{g}(u) \leq C$. Since the convergence in (11) is uniform, we obtain

$$
\begin{aligned}
\mathbb{P}\left(\sup _{(s, t) \in\left[0, f^{*}(u)\right] \times\left[0, g^{*}(u)\right]} X(s, t) \leq u\right) & =\mathbb{P}\left(\sup _{(s, t) \in[0, \bar{f}(u) \sqrt{m(u)}] \times[0, \bar{g}(u) \sqrt{m(u)}]} X(s, t) \leq u\right) \\
& \rightarrow \mathrm{e}^{-a b}
\end{aligned}
$$

as $u \rightarrow \infty$. On the other hand, by (12),

$$
\mathbb{P}\left(\sup _{(s, t) \in[0,1]^{2}} X(s, t) \leq u\right)^{f^{*}(u) g^{*}(u)}=\mathbb{P}\left(\sup _{(s, t) \in[0,1]^{2}} X(s, t) \leq u\right)^{\bar{f}(u) \bar{g}(u) \cdot m(u)} \rightarrow \mathrm{e}^{-a b},
$$

as $u \rightarrow \infty$, which gives (10).

Proof of (ii). Let us consider the case where $r>0$. Note that for $\boldsymbol{W}_{r}=\exp (2 \sqrt{r} \mathcal{W}-2 r)$ it holds that

$$
\begin{aligned}
\operatorname{var}\left(\exp \left(-\boldsymbol{W}_{r}\right)\right) & =\mathbb{E}\left(\exp \left(-2 \boldsymbol{W}_{r}\right)\right)-\mathbb{E}\left(\exp \left(-\boldsymbol{W}_{r}\right)\right)^{2} \\
& =\mathbb{P}\left(\max _{j \leq 2\lfloor\sqrt{m(u)}\rfloor, k \leq\lfloor\sqrt{m(u)}\rfloor} \tilde{X}_{j, k} \leq u\right)-\mathbb{P}\left(\max _{j, k \leq\lfloor\sqrt{m(u)}\rfloor} \tilde{X}_{j, k} \leq u\right)^{2}+o(1),
\end{aligned}
$$

due to Theorem 2. By contradiction, assume that the extremal index exists and equals $\theta \in(0,1]$. Then for any sequence $\left(z_{n}\right) \subset \mathbb{R}$ we have

$$
\begin{aligned}
& \mathbb{P}\left(\max _{j \leq\left\lfloor 2 \sqrt{m\left(z_{n}\right)}\right\rfloor, k \leq\left\lfloor\sqrt{m\left(z_{n}\right)}\right\rfloor} \tilde{X}_{j, k} \leq z_{n}\right)-\mathbb{P}\left(\max _{j, k \leq\left\lfloor\sqrt{m\left(z_{n}\right)}\right\rfloor} \tilde{X}_{j, k} \leq z_{n}\right)^{2} \\
&=\left(\mathbb{P}\left(\max _{j \leq 2\left\lfloor\sqrt{m\left(z_{n}\right)}\right\rfloor, k \leq\left\lfloor\sqrt{m\left(z_{n}\right)}\right\rfloor} \tilde{X}_{j, k} \leq z_{n}\right)-\mathbb{P}\left(\tilde{X}_{1,1} \leq z_{n}\right)^{2 m\left(z_{n}\right) \cdot \theta}\right) \\
&-\left(\mathbb{P}\left(\max _{j, k \leq\left\lfloor\sqrt{m\left(z_{n}\right)}\right\rfloor} \tilde{X}_{j, k} \leq z_{n}\right)^{2}-\left(\mathbb{P}\left(\tilde{X}_{1,1} \leq z_{n}\right)^{m\left(z_{n}\right) \cdot \theta}\right)^{2}\right) \\
&= o(1)
\end{aligned}
$$

as $n \rightarrow \infty$, which implies that $\operatorname{var}\left(\exp \left(-\mathcal{W}_{r}\right)\right)=0$. Since $r>0$ and $\mathcal{W}$ is an $N(0,1) \mathrm{RV}$ we obtain a contradiction. 


\section{Acknowledgements}

We would like to thank deeply the referees for comments and suggestions which improved the manuscript significantly. K. Dȩbicki was partially supported by NCN Grant Number 2011/01/B/ST1/01521 (2011-2013). The first two authors acknowledge kindly the partial support by the Swiss National Science Foundation Grant 200021-140633/1 and RARE -318984 (an FP7 Marie Curie IRSES Fellowship).

\section{References}

[1] Leadbetter, M. R., Lindgren, G. And Rootzén, H. (1983). Extremes and Related Properties of Random Sequences and Processes. Springer, New York.

[2] ArendarczyK, M. AND DȨBICKI, K. (2012). Exact asymptotics of supremum of a stationary Gaussian process over a random interval. Statist. Prob. Lett. 82, 645-652.

[3] TAN, Z. AND Hashorva, E. (2013). Limit theorems for extremes of strongly dependent cyclo-stationary $\chi$-processes. Etremes 16, 241-254.

[4] Pickands, J., III (1969). Upcrossing probabilities for stationary Gaussian processes. Trans. Amer. Math. Soc. 145, 51-73.

[5] Piterbarg, V. I. (1972). On the paper by J. Pickands 'Upcrossing probabilities for stationary Gaussian processes'. Vestnik Moskov. Univ. Ser. I Mat. Meh. 27, 25-30.

[6] Piterbarg, V. I. (1996). Asymptotic Methods in the Theory of Gaussian Processes and Fields ( Trans. Math. Monogr. 148). American Mathematical Society, Providence, RI.

[7] Bingham, N. H., Goldie, C. M. and Teugels, J. L. (1987). Regular Variation. Cambridge University Press.

[8] Embrechts, P., Klüppelberg, C. And Mikosch, T. (1997). Modelling Extremal Events for Insurance and Finance. Springer, Berlin.

[9] JaKubowski, A. (1991). Relative extremal index of two stationary processes. Stoch. Process. Appl. 37, 281-297.

[10] LeAdBetter, M. R. (1983). Extremes and local dependence in stationary sequences. Z. Wahrscheinlichkeitsch. 65, 291-306.

[11] O'Brien, G. L. (1987). Extreme values for stationary and Markov sequences. Ann. Prob. 15, $281-291$.

[12] French, J. P. And Davis, R. A. (2013). The asymptotic distribution of the maxima of a Gaussian random field on a lattice. Extremes 16, 1-26.

[13] Hsing, T. (1993). Extremal index estimation for a weakly dependent stationary sequence. Ann. Statist. 21, 2043-2071.

[14] Jakubowski, A. And Soja-KukieŁa, N. (2014). Managing local dependencies in limit theorems for maxima of stationary random fields. Submitted..

[15] Ferreira, H. (2006). The upcrossings index and the extremal index. J. Appl. Prob. 43, 927-937.

[16] Laurini, F. and Tawn, J. A. (2012). The extremal index for $\operatorname{GARCH}(1,1)$ processes. Extremes 15, 511-529.

[17] Dȩbicki, K., Hashorva, E. And Soja-KukieŁa, N. (2013). Extremes of homogeneous Gaussian random fields. Preprint. Available at http://arxiv.org/abs/1312.2863. 